International Journal of Mathematics 18 (2007) 281-299

\title{
Fractional Derivative as Fractional Power of Derivative
}

\author{
Vasily E. Tarasov \\ Skobeltsyn Institute of Nuclear Physics, \\ Moscow State University, Moscow 119991, Russia \\ E-mail: tarasov@theory.sinp.msu.ru
}

\begin{abstract}
Definitions of fractional derivatives as fractional powers of derivative operators are suggested. The Taylor series and Fourier series are used to define fractional power of self-adjoint derivative operator. The Fourier integrals and Weyl quantization procedure are applied to derive the definition of fractional derivative operator. Fractional generalization of concept of stability is considered.
\end{abstract}

MSC: 26A33 Fractional derivatives and integrals

\section{Introduction}

The theory of integrals and derivatives of non-integer order goes back to Leibniz, Liouville, Riemann, Grunwald, and Letnikov [1, 2]. Fractional analysis has found many applications in recent studies in mechanics and physics. The interest in fractional equations has been growing continually during the last few years because of numerous applications. In a short period of time the list of applications becomes long. For example, it includes the chaotic dynamics [3, 4], material sciences [5, 6, 7, 8, mechanics of fractal and complex media [9, 10, 11], quantum mechanics [12, 13, physical kinetics [3, 14, 15, 16], plasma physics [17, 20], electromagnetic theory [18, 19, 20], astrophysics [21], long-range dissipation [22, 26], nonHamiltonian mechanics [23, 24, 25], long-range interaction [28, 29, 30].

It is known that we can define a fractional power of operator [33, 34, 35, 31, 32, 36, 37. The integer power of operator can be easily realized. Therefore, we can realize the fractional power as the integer power series. In this paper, we use equations that represent the fractional power as a series of integer powers series. This representation allows us to define the fractional power of operator as a series of integer powers of operator. As a result, we obtain the definition of fractional derivatives as a fractional power of derivative operator.

Note that the well-known Riemann-Liouville fractional derivative can be represented as a power series of derivatives of integer order [1]:

$$
D_{a+}^{\alpha}=\sum_{n=0}^{\infty} A_{n}(x, a, \alpha) \frac{d^{n}}{d x^{n}}
$$


where

$$
A_{n}(x, a, \alpha)=\frac{(-1)^{n-1} \alpha \Gamma(n-\alpha)}{\Gamma(1-\alpha) \Gamma(n+1)} \frac{(x-a)^{n-\alpha}}{\Gamma(n+1-\alpha)}
$$

for the functions that are analytical in the interval $(a, b)$.

In Sec. 2, we point out some well-known definitions of functions of bounded and unbounded operators. In Sec. 3, the fractional derivatives are defined as fractional powers of coordinates that considered as Taylor series. In Sec. 4, the fractional derivatives are considered as fractional powers of coordinates that described as Fourier series for the interval. In Sec. 5, the fractional derivatives are defined as fractional powers of coordinates by using the Fourier integrals. In Sec. 6, the fractional derivatives are defined as fractional powers of coordinates by using the Weyl quantization. In Sec. 7, using fractional derivatives, we define the stability with respect to fractional variations.

\section{$2 \quad$ Function of bounded and unbounded operators}

Let us point out some well-known definitions of functions of bounded and unbounded operators [31, 32, 33, 34, 35].

\subsection{Power series}

Let us consider a bounded linear operator $A$ that is defined on the linear space $E$, and $A \in L(E, E)$, where $L(E, E)$ is a space of linear maps of $E$. Suppose the function $f(x)$ is an analytical function of the variable $x$ such that it can be represented as a power series

$$
f(x)=\sum_{n=0}^{\infty} f_{n} x^{n}
$$

Then, we can define

$$
f(A)=\sum_{n=0}^{\infty} f_{n} A^{n} .
$$

The operator $f(A)$ is a linear bounded operator $A$ on space $E$. For example, the exponential function of operator is defined by

$$
e^{A}=\sum_{n=0}^{\infty} \frac{1}{n !} A^{n}
$$

\subsection{Cauchy's integral formula}

The definition of operator function by power series can be generalized for wider class of functions. To realize this generalization, we use Cauchy's integral formula instead of power 
series. Cauchy's integral formula states that

$$
f\left(z_{0}\right)=\frac{1}{2 \pi i} \oint_{\Gamma} \frac{f(z) d z}{z-z_{0}}
$$

where the integral is a contour integral along the contour $\Gamma$ enclosing the point $z_{0}$.

We can define the algebraic isomorphism between an operator algebra and some functions [34. The function $f(z)=z$ corresponds to the operator $A$. The function $f\left(z-z_{0}\right)=$ $\left(z-z_{0}\right)^{-1}$ corresponds to the resolvent operator $R(z, A)=(z I-A)^{-1}$. If $|z|>r_{A}$, where $r_{A}$ is a spectral radius:

$$
r_{A}=\lim _{n \rightarrow \infty} \sqrt[n]{\left\|A^{n}\right\|}
$$

then the resolvent operator exists. The function of linear bounded operator is defined by

$$
f(A)=\frac{1}{2 \pi i} \oint_{\Gamma} f(z) R(z, A) d z,
$$

where

$$
R(z, A)=(z I-A)^{-1}, \quad z \in \rho(A) .
$$

Here $\Gamma=\partial G \in \sigma(A)$, where $\sigma(A)$ is a spectrum of operator $A$, and $\rho(A) \subset G$. For example, we can define the operator

$$
e^{A t}=\sum_{n=0}^{\infty} \frac{t^{n}}{n !} A^{n}=\frac{1}{2 \pi i} \oint_{\partial G} e^{z t} R(z, A) d z
$$

that is corresponded to the function $\exp (z t)$, and $\sigma(A) \subset G$.

As the second example, the operator $E_{z}$ that corresponds to the Heaviside function $\theta\left(z-z_{0}\right)$, where $\theta\left(z-z_{0}\right)=0$ for $z_{0} \geq z$, and $\theta\left(z-z_{0}\right)=1$ for $z_{0}<z$ is defined by

$$
E_{z}=E(z, A)=\frac{1}{2 \pi i} \oint_{\Gamma} \theta\left(z-z_{0}\right) R\left(z_{0}, A\right) d z_{0}
$$

and is called the spectral operator. The operator $E_{z}$ can be denoted by $\theta(z I-A)$.

\subsection{Spectral representation of self-adjoint unbounded operator}

It is known that spectral function $E_{z}$ exists for all self-adjoint operators $A$, and

$$
A x=\int_{-\infty}^{+\infty} z d E_{z} x
$$

where

$$
\|A x\|^{2}=\int_{-\infty}^{+\infty}|z|^{2} d\left(E_{z} x, x\right)<\infty .
$$


Then, the function $f(A)$ of self-adjoint operator $A$ can be defined by the equation

$$
f(A) x=\int_{-\infty}^{+\infty} f(z) d E_{z} x .
$$

As operator $A$, we can consider the self-adjoint derivative $-i \partial / \partial x$. For the father information about this approach, we can use Refs. [31, 32, 35, 45, 46].

\section{Fractional Derivatives by Taylor Series}

\subsection{Definition of Taylor series}

A one-dimensional Taylor series, which is an expansion of a real-valued function $f(x)$ about a point $x=a$, is given by

$$
f(x)=\sum_{n=0}^{\infty} f_{n}(x-a)^{n}
$$

where

$$
f_{n}=\frac{1}{n !} f^{(n)}(a),
$$

and $f^{(n)}(a)$ is the nth derivative of $f(x)$ evaluated at the point $x=a$.

Suppose the function $f(x)$ has all derivatives in the interval $|x-a|<a_{0}$, and the condition

$$
\lim _{n \rightarrow \infty} \frac{f^{(n)}(a)}{n !}(x-a)^{n}=0
$$

is satisfied, then the series

$$
f(x)=\sum_{n=0}^{\infty} \frac{f^{(n)}(a)}{n !}(x-a)^{n}
$$

converges to the function $f(x)$ for all intervals $|x-a|<a^{\prime}$, where $a^{\prime}<a_{0}$. This representation of the functions can be used to define fractional power of operator.

\subsection{Taylor series for fractional power of coordinate}

The Taylor series for fractional power of coordinate $f(x)=x^{\alpha}$ about a point $x=a>0$ is

$$
x^{\alpha}=\sum_{n=0}^{\infty} f_{n}(x-a)^{n}
$$

where

$$
f_{n}=\frac{1}{n !}\left(x^{\alpha}\right)^{(n)}(a)=\frac{B(\alpha, n)}{n ! a^{n-\alpha}},
$$




$$
B(\alpha, n)=\alpha(\alpha-1)(\alpha-2) \ldots(\alpha-n+1) .
$$

If $m-1<\alpha<m$, then

$$
B(\alpha, n)=(-1)^{n-m} \frac{(\alpha+1-m)_{m}}{(m-\alpha)_{n-m}}=(-1)^{n-m} \frac{\Gamma(n-\alpha) \Gamma(\alpha+1)}{\Gamma(m-\alpha) \Gamma(\alpha+1-m)},
$$

where

$$
(z)_{m}=z(z+1) \ldots(z+m-1) .
$$

For $0<\alpha<1$,

$$
B(\alpha, n)=(-1)^{n-1} \alpha(1-\alpha)_{n-1}=(-1)^{n-1} \frac{\Gamma(n-\alpha)}{\Gamma(1-\alpha)} .
$$

Using

$$
(x-a)^{n}=\sum_{k=0}^{n}(-1)^{n-k}\left(\begin{array}{l}
n \\
k
\end{array}\right) x^{k} a^{n-k}, \quad\left(\begin{array}{l}
n \\
k
\end{array}\right)=\frac{n !}{(n-k) ! k !},
$$

we rewrite Eq. (11) in the form

$$
x^{\alpha}=\sum_{n=0}^{\infty} \sum_{k=0}^{n} C(n, k, \alpha, a) x^{k}
$$

where

$$
C(n, \alpha, a)=\frac{(-1)^{k+1} a^{\alpha-k}}{(n-k) ! k !} \frac{\Gamma(n-\alpha)}{\Gamma(1-\alpha)} .
$$

Equation (14) represents the fractional power of coordinate as a series of integer powers. This representation allows us to define the fractional power of operator as a series of integer powers of operator.

It is known that self-adjoint operators have the real eigenvalues. Using Eq. (14) with $a>0$, we can define the fractional power of the self-adjoint operator $A$ by

$$
A^{\alpha}=\sum_{n=0}^{\infty} \frac{B(\alpha, n)}{n ! a^{n-\alpha}} A^{n} .
$$

For the operator $A=-i \partial / \partial x$, we have

$$
\left(-i \frac{d}{d x}\right)^{\alpha}=\sum_{n=0}^{\infty} \frac{B(\alpha, n)}{n ! a^{n-\alpha}}\left(-i \frac{d}{d x}-a\right)^{n},
$$

or, in the equivalent form

$$
\left(-i \frac{d}{d x}\right)^{\alpha}=\sum_{n=0}^{\infty} \sum_{k=0}^{n}(-i)^{k} C(n, k, \alpha, a) \frac{d^{k}}{d x^{k}} .
$$

As a result, we get that fractional derivative is defined as a series of integer powers of self-adjoint derivative operator. 


\subsection{Examples of computation of fractional derivatives}

Let us consider the fractional derivative (16) of constant $c$ :

$$
\left(-i \frac{d}{d x}\right)^{\alpha} c=\sum_{n=0}^{\infty} \frac{A(\alpha, n)}{n ! a^{n-\alpha}}\left(-i \frac{d}{d x}-a\right)^{n} c .
$$

Using

$$
\left(-i \frac{d}{d x}-a\right)^{n} c=(-a)^{n} c
$$

we get

$$
\left(-i \frac{d}{d x}\right)^{\alpha} c=a^{\alpha} c \sum_{n=0}^{\infty}(-1)^{n} \frac{B(\alpha, n)}{n !}
$$

If $0<\alpha<1$, then

$$
\left(-i \frac{d}{d x}\right)^{\alpha} c=-a^{\alpha} c \sum_{n=0}^{\infty} \frac{\Gamma(n-\alpha)}{\Gamma(n+1) \Gamma(1-\alpha)} .
$$

Let us consider the fractional derivative of a power $x^{m}$. From (17),

$$
\left(-i \frac{d}{d x}\right)^{\alpha} x^{m}=\sum_{n=0}^{\infty} \sum_{k=0}^{n}(-i)^{k} C(n, k, \alpha, a)\left(x^{m}\right)^{(k)} .
$$

Using

$$
\left(x^{m}\right)^{(k)}=m(m-1) \ldots(m-k+1) x^{m-k}=\frac{m !}{(m-k) !} x^{m-k}
$$

for $k \leq m$, and $\left(x^{m}\right)^{(k)}=0$ for $k>m$, we obtain

$$
\left(-i \frac{d}{d x}\right)^{\alpha} x^{m}=\sum_{n=0}^{m} \sum_{k=0}^{n}(-i)^{k} C(n, k, \alpha, a) \frac{m !}{(m-k) !} x^{m-k}
$$

\section{Fractional Derivatives by Fourier Series}

\subsection{Fourier series}

Fourier series of a function $f(x) \in L_{2}[-l, l]$ is an expansion in terms of an infinite sum of sines and cosines. Since sines and cosines form a complete orthogonal system over $[-l, l]$, the Fourier series is given by

$$
f(x)=\frac{a_{0}}{2}+\sum_{n=1}^{\infty}\left[a_{n} \cos \left(\frac{\pi n x}{l}\right)+b_{n} \sin \left(\frac{\pi n x}{l}\right)\right]
$$


where

$$
a_{n}=\frac{1}{l} \int_{-l}^{+l} f(x) \cos \left(\frac{\pi n x}{l}\right) d x, \quad b_{n}=\frac{1}{l} \int_{-l}^{+l} f(x) \sin \left(\frac{\pi n x}{l}\right) d x
$$

and $n$ is a positive integer number.

Let us consider the Fourier series for $f(x)=|x|^{\alpha} \in L^{2}[-l ; l]$, where $\alpha$ is a positive fractional power. The Fourier series of this function for $x \in[-l,+l]$ is

$$
|x|^{\alpha}=\frac{a_{0}}{2}+\sum_{k=1}^{\infty} a_{k} \cos \left(\frac{\pi k x}{l}\right)
$$

where

$$
a_{k}=\frac{1}{l} \int_{-l}^{l}|y|^{\alpha} \cos \left(\frac{\pi k y}{l}\right) d y=\frac{2}{l} \int_{0}^{l} y^{\alpha} \cos \left(\frac{\pi k y}{l}\right) d y, \quad k=0,1,2, \ldots
$$

The cosine can be represented as a power series. Therefore, equation (26) allows us to present the fractional power as a series of integer powers series. Then, we can define fractional derivative on the interval $[-l, l]$ as a fractional power of derivative.

\subsection{Fractional derivative}

The fractional power of derivative operator for the interval $[-l ; l]$, we can define by

$$
\left(-i \frac{d}{d x}\right)^{\alpha}=\frac{a_{0}}{2}+\sum_{k=1}^{\infty} a_{k} \cos \left(-i \frac{\pi k}{l} \frac{d}{d x}\right)
$$

It is known that we can $\operatorname{define} \exp (A)$ for the self-adjoint operator $A=-i d / d x$ by

$$
e^{-i d / d x}=\sum_{n=0}^{\infty} \frac{1}{n !} A^{n}=\sum_{n=0}^{\infty} \frac{(-i)^{n}}{n !}\left(\frac{d}{d x}\right)^{n} .
$$

Using

$$
\begin{gathered}
\cos (A)=\frac{1}{2}\left(e^{i A}+e^{-i A}\right)=\frac{1}{2}\left(\sum_{n=0}^{\infty} \frac{(i A)^{n}}{n !}+\sum_{n=0}^{\infty} \frac{(-i A)^{n}}{n !}\right)= \\
=\sum_{n=0}^{\infty} \frac{1}{2(n !)}\left[(A)^{n}+(-A)^{n}\right]=\sum_{n=0}^{\infty} \frac{1+(-1)^{n}}{2(n !)} A^{n}=\sum_{m=0}^{\infty} \frac{1}{(2 m) !} A^{2 m}
\end{gathered}
$$

we have

$$
\cos \left(-i \frac{d}{d x}\right)=\sum_{m=0}^{\infty} \frac{1}{(2 m) !}\left(\frac{d}{d x}\right)^{2 m}
$$


Equation (67) and (29) allows us to define the fractional power of operator as a series of integer powers of operator. Using (29), we rewrite (27) as

$$
\left(-i \frac{d}{d x}\right)^{\alpha}=\frac{a_{0}}{2}+\sum_{k=1}^{\infty} a_{k} \sum_{m=0}^{\infty} \frac{1}{(2 m) !}\left(\frac{\pi k}{l}\right)^{2 m}\left(\frac{d}{d x}\right)^{2 m}
$$

or by the equivalent equation

$$
\left(-i \frac{d}{d x}\right)^{\alpha}=\frac{a_{0}}{2}+\sum_{m=0}^{\infty} S_{m}\left(\frac{d}{d x}\right)^{2 m}
$$

where

$$
S_{m}=\frac{1}{(2 m) !} \sum_{k=1}^{\infty}\left(\frac{\pi k}{l}\right)^{2 m} a_{k}
$$

Let us compute the coefficients $a_{k}$ by

$$
a_{k}=\frac{1}{l^{\alpha}} \int_{0}^{l} y^{\alpha} \cos (\pi k y) d y
$$

As a result, we obtain

$$
\begin{gathered}
a_{k}=2^{\alpha} \pi^{-1 / 2-\alpha} l^{-\alpha} k^{-1-\alpha}\left(\frac{2^{-\alpha} \pi^{-1 / 2+\alpha} k^{\alpha}(\alpha+3) \sin (\pi k)}{(\alpha+1)(\alpha+3)}+\right. \\
+\frac{2^{-\alpha} \pi^{-1 / 2+\alpha} k^{\alpha}[\pi k \cos (\pi k)-\sin (\pi k)]}{\alpha+1}+\frac{2^{-\alpha} \sqrt{k} \alpha L(\alpha+1 / 2,3 / 2, \pi k) \sin (\pi k)}{\alpha+1}- \\
\left.-\frac{2^{-\alpha}[\pi k \cos (\pi k)-\sin (\pi k)] L(\alpha+3 / 2,1 / 2, \pi k)}{\sqrt{k} \pi(\alpha+1)}\right),
\end{gathered}
$$

where $L(\mu, \nu, z)$ is the Lommel function [38].

\subsection{Complex Fourier series}

The real-valued function $f(x)$, which is defined on $[-\mathrm{L} / 2, \mathrm{~L} / 2]$, can be presented by

$$
f(x)=\sum_{n=-\infty}^{\infty} f_{n} e^{i(2 \pi n / L) x}
$$

where

$$
f_{n}=\frac{1}{L} \int_{-L / 2}^{+L / 2} f(x) e^{-i(2 \pi n x / L)} d x
$$


The operator function $f(A)$ is defined by

$$
f(A)=\sum_{n=-\infty}^{\infty} f_{n} e^{i(2 \pi n / L) A}
$$

where $f_{n}$ are defined in (34), and

$$
e^{i(2 \pi n / L) A}=\sum_{k=0}^{\infty} i^{k} \frac{(2 \pi n / L)^{k}}{k !} A^{k} .
$$

Substitution of (36) into (35) gives

$$
f(A)=\sum_{n=-\infty}^{\infty} \sum_{k=0}^{\infty} f_{n} \frac{(i 2 \pi n / L)^{k}}{k !} A^{k} .
$$

Then the fractional power of self-adjoint derivative on $[-L / 2,+L / 2]$ can be presented by

$$
\left(-i \frac{d}{d x}\right)^{\alpha}=\sum_{n=-\infty}^{\infty} \sum_{k=0}^{\infty} f(n, k)\left(\frac{d}{d x}\right)^{k}
$$

where

$$
f(n, k)=\frac{(2 \pi n)^{k}}{k ! L^{k+1}} \int_{+L / 2}^{-L / 2}|x|^{\alpha} \cos (n x) d x .
$$

Equation (38) represents the fractional power as a series of integer powers. This representation defines the fractional power of derivative as a series with integer powers of derivatives.

\section{Fourier Transform and Fourier Integral}

\subsection{Fractional derivative by Fourier integral}

Let us consider a function $f(x)$ with $n$ variables $x$. Suppose $A_{1}, A_{2}, \ldots, A_{n}$ are $n$ elements of commutative operator algebra $\mathcal{A}$. For example, $\mathcal{A}$ is an algebra of operators in linear space. We denote by $\tilde{f}$ the Fourier transform for $f(x)$ :

$$
\tilde{f}(y)=\int_{-\infty}^{+\infty} f(x) e^{-i x y} d x, \quad x y=x_{1} y_{1}+\ldots+x_{n} y_{n}
$$

where

$$
f(x)=\frac{1}{(2 \pi)^{n}} \int_{-\infty}^{+\infty} \tilde{f}(y) e^{i\left(y_{1} x_{1}+\ldots+y_{n} x_{n}\right)} d y=\frac{1}{(2 \pi)^{n}} \int_{-\infty}^{+\infty} d y \tilde{f}(y) e^{i y A}
$$


The operator function $f(A)$ of elements $A_{1}, \ldots, A_{n}$ is defined by

$$
f(A)=\frac{1}{(2 \pi)^{n}} \int_{-\infty}^{+\infty} \tilde{f}(y) e^{i\left(y_{1} A_{1}+\ldots+y_{n} A_{n}\right)} d y=\frac{1}{(2 \pi)^{n}} \int_{-\infty}^{+\infty} d y \tilde{f}(y) e^{i y A} .
$$

Substitution of Eq. (40) into Eq. (42) gives

$$
f(A)=\frac{1}{(2 \pi)^{n}} \int_{-\infty}^{+\infty} \tilde{f}(y) e^{i y A} d y=\frac{1}{(2 \pi)^{n}} \int_{-\infty}^{+\infty} d y \int_{-\infty}^{+\infty} d x f(x) e^{i y(A-x)} .
$$

In general, we must have the exact definition of these integrals and description of possible class of symbols and algebras of elements $A_{1}, . ., A_{n}$.

To define the fractional power of the operator $A$, we use (42) in the form

$$
A^{\alpha}=\frac{1}{(2 \pi)^{n}} \int_{-\infty}^{+\infty} d x \int_{-\infty}^{+\infty} d y|x|^{\alpha} e^{i y(A-x)}
$$

This equation can be considered as a definition of fractional power of operator A.

For $f(x)=|x|^{\alpha}$, where $\alpha \neq-1,-3, \ldots$, we have [39]:

$$
\tilde{f}(y)=\int_{-\infty}^{+\infty} d x|x|^{\alpha} e^{-i x y}=-2 \sin (\pi \alpha / 2) \Gamma(\alpha+1)|y|^{-\alpha-1} .
$$

Then Eq. (44) gives

$$
A^{\alpha}=-\frac{2 \sin (\pi \alpha / 2) \Gamma(\alpha+1)}{(2 \pi)^{n}} \int_{-\infty}^{+\infty} d y|y|^{-\alpha-1} e^{i y A}
$$

For the self-adjoint derivative operators

$$
A_{1}=-i \frac{\partial}{\partial x_{1}}, . ., A_{n}=-i \frac{\partial}{\partial x_{n}}
$$

equation (45) is

$$
\left(-i \frac{\partial}{\partial x}\right)^{\alpha}=-\frac{2 \sin (\pi \alpha / 2) \Gamma(\alpha+1)}{(2 \pi)^{n}} \int_{-\infty}^{+\infty} d y|y|^{-\alpha-1} \exp \left(y \frac{\partial}{\partial x}\right) .
$$

As a result, we obtain the fractional derivative operator as a fractional power of self-adjoint derivative operator. 


\subsection{Fractional power of self-adjoint derivative operator}

Let us consider the self-adjoint derivative operators

$$
D_{x}=-i \frac{\partial}{\partial x}=\left(-i \frac{\partial}{\partial x_{1}}, \ldots,-i \frac{\partial}{\partial x_{n}}\right)
$$

It is easy to prove that $f(p)=p^{\alpha} \in S^{\infty}\left(\mathbb{R}_{p}^{n}\right)$. Here, $S^{\infty}$ is the space of symbols that are slowly growth on the infinity. This space is defined as

$$
S^{\infty}\left(\mathbb{R}^{n}\right)=\cup_{l} \cap_{k} S_{l}^{k}\left(\mathbb{R}^{n}\right),
$$

where $S_{l}^{k}\left(\mathbb{R}^{n}\right)$ is a space of functions from the class $C^{k}\left(\mathbb{R}^{n}\right)$ with the norm

$$
\|f\|_{S_{l}^{k}\left(\mathbb{R}^{n}\right)}=\sup _{\mathbb{R}^{n}}\left(1+|x|^{2}\right)^{l / 2}\left(\sum_{|a|}\left\|f^{(a)}(x)\right\|\right) .
$$

The space $S^{\infty}\left(\mathbb{R}^{n}\right)$ is defined by

$$
\exists r, \quad \forall s \quad\|f\|_{r, s}=\sup _{x}\left\{(1+|x|)^{r}\left|\left(\frac{\partial}{\partial x}\right)^{s} f(x)\right|\right\}<\infty .
$$

The fractional powers of the operator (47) are elements of algebra $\mathcal{L}\left(S^{\infty}, S^{\infty}\right)$ of all continuous linear maps of the space $S^{\infty}\left(\mathbb{R}^{n}\right)$. Then, the fractional derivative operator

$$
D_{x}^{\alpha}=\left(-i \frac{\partial}{\partial x}\right)^{\alpha}
$$

is a fractional power of self-adjoint derivative operator. The operator (48) acts on the arbitrary function $u(x) \in C^{\infty}\left(\mathbb{R}^{n}\right)$ by

$$
\left(-i \frac{\partial}{\partial x}\right)^{\alpha} u(x)=\tilde{F}_{p \rightarrow x} p^{\alpha} F_{y \rightarrow p} u(y),
$$

where

$$
F_{y \rightarrow p} u(y)=\left(\frac{1}{2 \pi i}\right)^{n / 2} \int_{-\infty}^{+\infty} e^{i p y} u(y) d y,
$$

is the direct Fourier transform, and

$$
\tilde{F}_{p \rightarrow x} \Psi(p)=\left(\frac{1}{2 \pi i}\right)^{n / 2} \int_{-\infty}^{+\infty} e^{-i p x} \Psi(p) d p
$$

is the Fourier transform. 
Proposition. The operator

$$
D_{x}^{\alpha}=\left(-i \frac{\partial}{\partial x}\right)^{\alpha}
$$

has the symbol

$$
\operatorname{symb}\left\{D_{x}^{\alpha}\right\}(p)=p^{\alpha} .
$$

It is not hard to prove this proposition. Using Eq. (49), we get

$$
\begin{aligned}
D_{x}^{\alpha} e_{p}(x)=\left(-i \frac{\partial}{\partial x}\right)^{\alpha} e_{p}(x)=\left(-i \frac{\partial}{\partial x}\right)^{\alpha} e^{i p x}=\tilde{F}_{z \rightarrow x} z^{\alpha} F_{y \rightarrow z} e^{i p y}= \\
=\tilde{F}_{z \rightarrow x}\left(z^{\alpha}(2 \pi i)^{n / 2} \delta(z-p)\right)= \\
=\tilde{F}_{z \rightarrow x}\left(p^{\alpha}(2 \pi i)^{n / 2} \delta(z-p)\right)=p^{\alpha} e^{i p x}=p^{\alpha} e_{p}(x) .
\end{aligned}
$$

As a result, we obtain

$$
D_{x}^{\alpha} e_{p}(x)=p^{\alpha} e_{p}(x) .
$$

Multiplying both sides of (54) on $e_{-p}(x)$, we get $e_{-p}(x) D_{x}^{\alpha} e_{p}(x)=p^{\alpha}$ that proves (53).

\section{Fractional Derivative by Quantization Map}

\subsection{Quantization procedure for coordinate representation}

Let us consider the quantum mechanics in coordinate representation. It is known that quantization $\hat{Q}$ is a linear map of coordinate $q$ and momentum $p$ into self-adjoint operators

$$
\hat{Q}(q)=\hat{q}=q, \quad \hat{Q}(p)=\hat{p}=-i \hbar \frac{\partial}{\partial q}, \quad \hat{Q}(1)=\hat{I} .
$$

Using linearity of quantization map [40, 41], we get

$$
\hat{Q}(a q+b p)=a \hat{q}+b \hat{p} .
$$

Obviously, we have

$$
\hat{Q}\left([a q+b p]^{n}\right)=[a \hat{q}+b \hat{p}]^{n} .
$$

Using the power series

$$
\exp (x)=\sum_{n=0}^{\infty} \frac{1}{n !} x^{n},
$$

we get

$$
\hat{Q}(\exp [(i / \hbar)(a q+b p)])=\exp [(i / \hbar)(a \hat{q}+b \hat{p})] .
$$

This allows us to define the function of operators $\hat{q}$ and $\hat{p}$ by using the Fourier transforms [40, 41, 42, 43, 44, 45, 46]. 


\subsection{Weyl quantization}

Canonical quantization defines a map of real-valued functions into self-adjoint operators [40, 41, 42, 43, 44, 45, 46]. A classical observable is described by some real-valued function $A(q, p)$ from a function space $\mathcal{M}$. Quantization of this function leads to self-adjoint operator $\hat{A}(\hat{q}, \hat{p})$ from some operator space $\hat{\mathcal{M}}$.

Let us consider main points of the usual method of canonical quantization [40, 42, 45, 46]. Suppose $q_{k}$ are canonical coordinates and $p_{k}$ are canonical momenta, where $k=1, \ldots, n$. The basis of the space $\mathcal{M}$ of functions $A(q, p)$ is defined by functions

$$
W(a, b, q, p)=e^{(i / \hbar)(a q+b p)}, \quad a q=\sum_{k=1}^{n} a_{k} q_{k} .
$$

Quantization transforms coordinates $q_{k}$ and momenta $p_{k}$ to operators $\hat{q}_{k}$ and $\hat{p}_{k}$. Weyl quantization of the basis functions (58) leads to the Weyl operators

$$
\hat{Q}(W(a, b, q, p))=\hat{W}(a, b, \hat{q}, \hat{p})=e^{(i / \hbar)(a \hat{q}+b \hat{p})}, \quad a \hat{q}=\sum_{k=1}^{n} a_{k} \hat{q}_{k} .
$$

Operators (59) form a basis of the operator space $\hat{\mathcal{M}}$. Classical observable, which is characterized by the function $A(q, p)$, can be represented in the form

$$
A(q, p)=\frac{1}{(2 \pi \hbar)^{n}} \int_{-\infty}^{+\infty} \tilde{A}(a, b) W(a, b, q, p) d^{n} a d^{n} b
$$

where

$$
\tilde{A}(a, b)=\frac{1}{(2 \pi \hbar)^{n}} \int_{-\infty}^{+\infty} \tilde{A}(a, b) W(a, b, q, p) d^{n} a d^{n} b
$$

i.e., $\tilde{A}(a, b)$ is the Fourier image of the function $A(q, p)$. Quantum observable $\hat{A}(\hat{q}, \hat{p})$, which corresponds to $A(q, p)$, is

$$
\hat{Q}(A(q, p))=\hat{A}(\hat{q}, \hat{p})=\frac{1}{(2 \pi \hbar)^{n}} \int_{-\infty}^{+\infty} \tilde{A}(a, b) \hat{W}(a, b, \hat{q}, \hat{p}) d^{n} a d^{n} b .
$$

This formula can be considered as an operator expansion for $\hat{A}(\hat{q}, \hat{p})$ in the operator basis (59). Substitution of (61) into (62) gives

$$
\hat{A}(\hat{q}, \hat{p})=\frac{1}{(2 \pi \hbar)^{2 n}} \int_{-\infty}^{+\infty} d^{n} a d^{n} b \int_{-\infty}^{+\infty} d^{n} q d^{n} p A(q, p) \hat{W}(a, b, \hat{q}-q \hat{I}, \hat{p}-p \hat{I}) .
$$

The function $A(q, p)$ is called the Weyl symbol of the operator $\hat{A}(\hat{q}, \hat{p})$. Canonical quantization defined by (63) is called the Weyl quantization. The Weyl operator (59) in formula (63) leads to the Weyl quantization. Another basis operator leads to different quantization scheme [42]. 


\subsection{Fractional derivative by Weyl quantization map}

Let us consider a quantization map of real-valued function $f(p)=|p|^{\alpha}$ into self-adjoint operator. Quantization of this function leads to some self-adjoint operator $\hat{f}(\hat{p})$ from the operator space $\hat{\mathcal{M}}_{p}$, where $\hat{p}_{k}=-i \partial / \partial x_{k}$ and $k=1, \ldots, n$. Then

$$
D_{x}^{\alpha}=f(\hat{p})=\hat{p}^{\alpha}=\left(-i \partial / \partial x_{k}\right)^{\alpha} .
$$

The basis of the space $\mathcal{M}_{p}$ is defined by functions

$$
W(a, p)=e^{i b p}, \quad a p=\sum_{k=1}^{n} a_{k} p_{k} .
$$

Quantization maps $p_{k}$ into $\hat{p}_{k}=-i \partial / \partial x_{k}$. Weyl quantization of the functions (64) leads to

$$
\hat{Q}(W(a, b))=\hat{W}(a, \hat{p})=e^{i a \hat{p}}, \quad a \hat{p}=\sum_{k=1}^{n} a_{k} \hat{p}_{k} .
$$

The operators (65) form a basis of the operator space $\hat{\mathcal{M}}_{p}$. Using Fourier transform, the function $f(p)=|p|^{\alpha}$ can be presented by

$$
f(p)=\frac{1}{(2 \pi)^{n / 2}} \int_{-\infty}^{+\infty} \tilde{f}(a) W(a, p) d^{n} a,
$$

where

$$
\tilde{f}(a)=\frac{1}{(2 \pi)^{n / 2}} \int_{-\infty}^{+\infty} f(p) W(a, p) d^{n} p,
$$

i.e., $\tilde{f}(a)$ is the Fourier image of the function $f(p)$. Quantum observable $\hat{f}(\hat{p})$, which corresponds to $f(p)$, is defined by the formula

$$
\hat{Q}(f(p))=\hat{f}(\hat{p})=\frac{1}{(2 \pi)^{n / 2}} \int_{-\infty}^{+\infty} \tilde{f}(a) \hat{W}(a, \hat{p}) d^{n} a .
$$

This formula can be considered as an operator expansion for $\hat{f}(\hat{p})$ in the operator basis (59)). From (67) and (68), we obtain

$$
\hat{f}(\hat{p})=\frac{1}{(2 \pi)^{n}} \int_{-\infty}^{+\infty} d^{n} a \int_{-\infty}^{+\infty} d^{n} p f(p) \hat{W}(a, \hat{p}-p \hat{I}) .
$$

The function $f(p)$ is the Weyl symbol of the operator $\hat{f}(\hat{p})$.

For $f(p)=|p|^{\alpha}$, where $\alpha$ is a positive real number, we obtain

$$
D_{x}^{\alpha}=\left(-i \frac{d}{d x}\right)^{\alpha}=\frac{1}{(2 \pi)^{n}} \int_{-\infty}^{+\infty} d^{n} a \int_{-\infty}^{+\infty} d^{n} p|p|^{\alpha} \hat{W}(a, \hat{p}-p \hat{I}) .
$$

As a result, we have the definition of fractional derivatives on $\mathbb{R}^{n}$ as a fractional power of self-adjoint derivative. 


\section{$7 \quad$ Fractional Stability}

In this section, we use the fractional generalization of variations of variables. Fractional integrals and derivatives are used for stability problems [47, 48, 49, 50, 51]. In this paper, we consider the properties of dynamical systems with respect to fractional variations [25]. We formulate stability with respect to motion changes at fractional changes of variables. Some systems can be unstable "in sense of Lyapunov", and be stable with respect to fractional variations.

\subsection{Fractional variation derivative}

Let us consider dynamical system that is defined by the ordinary differential equations. Suppose that the motion of dynamical system is described by the equations

$$
\frac{d}{d t} y_{k}=F_{k}(y), \quad k=1, \ldots, n
$$

Here $y_{1}, \ldots, y_{n}$ be real variables that define the state of dynamical system.

Let us consider the variation $\delta y_{k}$ of variables $y_{k}$. The unperturbed motion is satisfied to zero value of variation $\delta y_{k}=0$. The variation describes that as function $f(y)$ changes at changes of argument $y$. The first variation describes changes of function with respect to the first power of changes of $y$ :

$$
\delta f(y)=D_{y}^{1} f(y) d y
$$

where

$$
D_{y}^{1} f(y)=\frac{\partial f(y)}{\partial y}
$$

The second variation describes changes of function with respect to the second power of changes of $y$ :

$$
\delta^{2} f(y)=D_{y}^{2} f(y)(d y)^{2} .
$$

The variation $\delta^{n}$ of integer order $n$ is defined by the derivative of integer order $D_{y}^{n} f(y)=$ $\partial^{n} f / \partial y^{n}$.

Let us define the variation of fractional order as a fractional exterior derivative of the function (zero-form) by the equation

$$
\delta^{\alpha} f=D_{y}^{\alpha} f(\delta y)^{\alpha}
$$

where $D_{y}^{\alpha}$ is a fractional derivative with respect to $y$.

The fractional variation of order $\alpha$ describes the function $f(y)$ changes with respect to fractional power of variable $y$ changes. The variation of fractional order is defined by the derivative of fractional order. 


\subsection{Equations for fractional variations}

Let us derive the equations for fractional variations $\delta^{\alpha} y_{k}$. We consider the fractional variation of equation (171) in the form:

$$
\delta^{\alpha} \frac{d}{d t} y_{k}=\delta^{\alpha} F_{k}(y), \quad k=1, \ldots, n
$$

Using the definition of fractional variation (74), we have

$$
\delta^{\alpha} F_{k}(y)=\left[D_{y_{l}}^{\alpha} F_{k}\right]\left(\delta y_{l}\right)^{\alpha}, \quad k=1, \ldots, n .
$$

From Eq. (176), and the property of variation

$$
\delta^{\alpha} \frac{d}{d t} y_{k}=\frac{d}{d t} \delta^{\alpha} y_{k}
$$

where $y_{k}=y_{k}(t, a)$, we obtain

$$
\frac{d}{d t} \delta^{\alpha} y_{k}=\left[D_{y_{l}}^{\alpha} F_{k}\right]\left(\delta y_{l}\right)^{\alpha}, \quad k=1, \ldots, n
$$

Note that in the left hand side of Eq. (78), we have fractional variation of $\delta^{\alpha} y_{k}$, and in the right hand side - fractional power of variation $\left(\delta y_{k}\right)^{\alpha}$.

Let us consider the fractional variation of the variable $y_{k}$. Using Eq. (174), we get

$$
\delta^{\alpha} y_{k}=\left[D_{y_{l}}^{\alpha} y_{k}\right]\left(\delta y_{l}\right)^{\alpha}, \quad k=1, \ldots, n
$$

For the Riemann-Liouville fractional derivative,

$$
D_{y_{l}}^{\alpha} y_{k} \neq 0
$$

if $k \neq l$. Therefore for simplification of our transformations, we use the fractional derivative as a fractional power of derivative

$$
D_{y_{l}}^{\alpha} y_{k}=\delta_{k l} D_{y_{l}}^{\alpha} y_{l}
$$

where $\delta_{k l}$ is a Kronecker symbol. Substituting Eq. (80) into Eq. (179), we can express the fractional power of variations $\left(\delta y_{k}\right)^{\alpha}$ through the fractional variation $\delta^{\alpha} y_{k}$ :

$$
\left(\delta y_{k}\right)^{\alpha}=D_{y_{k}}^{\alpha} y_{k} \delta^{\alpha} y_{k}
$$

Substitution of Eq. (81) into Eq. (178) gives

$$
\frac{d}{d t} \delta^{\alpha} y_{k}=\left[D_{y_{l}}^{\alpha} y_{l}\right]\left[D_{y_{l}}^{\alpha} F_{k}\right] \delta^{\alpha} y_{l}
$$


Here we mean the sum on the repeated index $l$ from 1 to $n$. Equation (82) is equations for fractional variations. Let us denote $x_{k}$ the fractional variations $\delta^{\alpha} y_{k}$ :

$$
x_{k}=\delta^{\alpha} y_{k}=\left[D_{y_{k}}^{\alpha} y_{k}\right]\left(\delta y_{k}\right)^{\alpha} .
$$

As a result, we obtain the differential equation for fractional variations

$$
\frac{d}{d t} x_{k}=a_{k l}(\alpha) x_{l}
$$

where

$$
a_{k l}(\alpha)=\left[D_{y_{l}}^{\alpha} y_{l}\right] D_{y_{l}}^{\alpha} F_{k}
$$

Using the matrix $X^{t}=\left(x_{1}, \ldots, x_{n}\right)$, and $A_{\alpha}=\left\|a_{k l}(\alpha)\right\|$, we can rewrite Eq. (84) in the matrix form

$$
\frac{d}{d t} X=A_{\alpha} X
$$

Equation (86) is a linear differential equation. To define the stability with respect to fractional variations, we consider the characteristic equation

$$
\operatorname{Det}\left[A_{\alpha}-\lambda E\right]=0
$$

with respect to $\lambda$. If the real part $R e\left[\lambda_{k}\right]$ of all eigenvalues $\lambda$ for the matrix $A_{\alpha}$ are negative, then the unperturbed motion is asymptotically stable with respect to fractional variations. If the real part $\operatorname{Re}\left[\lambda_{k}\right]$ of one of the eigenvalues $\lambda$ of the matrix $A_{\alpha}$ is positive, then the unperturbed motion is unstable with respect to fractional variations.

A system is said to be stable with respect to fractional variations if for every $\epsilon$, there is a $\delta_{0}$ such that:

$$
\left\|\delta^{\alpha} y\left(t_{0}\right)\right\|<\delta_{0} \quad=>\left\|\delta^{\alpha} y(\alpha, t)\right\|<\epsilon \quad \forall t \in \mathbb{R}_{+} .
$$

The dynamical system is said to be asymptotically stable with respect to fractional variations $\delta^{\alpha} y(t, \alpha)$ if as

$$
t \rightarrow \infty, \quad\left\|\delta^{\alpha} y(t, \alpha)\right\| \rightarrow 0 .
$$

The concept of stability with respect to fractional variations is wider than the usual Lyapunov or asymptotic stability. Fractional stability includes concept of "integer" stability as a special case $\alpha=1$. Some systems can be unstable with respect to first variation of

states, and be stable with respect to fractional variation. Therefore fractional derivatives expand our possibility to research the properties of dynamical systems.

\section{References}

[1] S.G. Samko, A.A. Kilbas, O.I. Marichev, Fractional Integrals and Derivatives Theory and Applications (Gordon and Breach, New York, 1993)

[2] K.B. Oldham, J. Spanier, The Fractional Calculus (Academic Press, New York, 1974) 
[3] G.M. Zaslavsky, "Chaos, fractional kinetics, and anomalous transport" Phys. Rep. 371 (2002) 461-580.

[4] G.M. Zaslavsky, Hamiltonian Chaos and Fractional Dynamics (Oxford University Press, Oxford, 2005)

[5] Applications of Fractional Calculus in Physics (World Scientific, Singapore, 2000)

[6] M. Caputo, Elasticita e Dissipazione (Zanichelli, Bologna, 1969) in Italian

[7] R.R. Nigmatullin, "The realization of the generalized transfer equation in a medium with fractal geometry" Phys. Status Solidi B 133 (1986) 425-430; "Fractional integral and its physical interpretation" Theor. Math. Phys. 90 (1992) 242-251.

[8] A. Le Mehaute, R.R. Nigmatullin, L. Nivanen, Fleches du temps et geometric fractale (Hermes, Paris, 1998) Chapter 5. in French

[9] A. Carpinteri, F. Mainardi, Fractals and Fractional Calculus in Continuum Mechanics (Springer, New York, 1997)

[10] V.E. Tarasov, "Continuous medium model for fractal media" Phys. Lett. A 336 (2005) 167-174; "Fractional Fokker-Planck equation for fractal media" Chaos 15 (2005) 023102; "Possible experimental test of continuous medium model for fractal media" Phys. Lett. A 341 (2005) 467-472; "Fractional hydrodynamic equations for fractal media" Ann. Phys. 318 (2005) 286-307; "Wave equation for fractal solid string" Mod. Phys. Lett. B 19 (2005) 721-728; "Dynamics of fractal solid" Int. J. Mod. Phys. B 19 (2005) 4103-4114; "Fractional Chapman-Kolmogorov equation" Mod. Phys. Lett. B 21 (2007) 163-174 (2007).

[11] V.E. Tarasov, G.M. Zaslavsky, "Fractional Ginzburg-Landau equation for fractal media" Physica A 354 (2005) 249-261.

[12] N. Laskin, "Fractional quantum mechanics" Phys. Rev. E 62 (2000) 3135-3145; "Fractional quantum mechanics and Levy path integrals" Phys. Lett. A 268 (2000) 298-305; "Fractals and quantum mechanics" Chaos 10 (2000) 780-790; "Fractional Schrodinger equation" Phys. Rev. E 66 (2002) 056108.

[13] M. Naber, "Time fractional Schrodinger equation" J. Math. Phys. 45 (2004) 3339-3352.

[14] G.M. Zaslavsky, "Fractional kinetic equation for Hamiltonian chaos" Physica D 76 (1994) 110-122.

[15] A.I. Saichev, G.M. Zaslavsky, "Fractional kinetic equations: solutions and applications" Chaos 7 (1997) 753-764.

[16] G.M. Zaslavsky, M.A. Edelman, "Fractional kinetics: from pseudochaotic dynamics to Maxwell's demon" Physica D 193 (2004) 128-147.

[17] B.A. Carreras, V.E. Lynch, G.M. Zaslavsky, "Anomalous diffusion and exit time distribution of particle tracers in plasma turbulence model" Physics of Plasmas 8 (2001) 5096-5103.

[18] J. Lutzen, "Liouville's differential calculus of arbitrary order and its electrodynamical origin," in Proc. 19th Nordic Congress Mathenzaticians, (Icelandic Mathematical Soc., Reykjavik, 1985) pp.149-160. 
[19] L.M. Zelenyi, A.V. Milovanov, "Fractal topology and strange kinetics: from percolation theory to problems in cosmic electrodynamics" Physics Uspekhi 47 (2004) 749-788.

[20] V.E. Tarasov, "Electromagnetic field of fractal distribution of charged particles" Physics of Plasmas 12 (2005) 082106; "Multipole moments of fractal distribution of charges" Mod. Phys. Lett. B 19 (2005) 1107-1118; "Magnetohydrodynamics of fractal media" Physics of Plasmas 13 (2006) 052107.

[21] V.E. Tarasov, "Gravitational field of fractal distribution of particles" Celes. Mech. Dynam. Astron. 19 (2006) 1-15.

[22] F. Mainardi, R. Gorenflo, "On Mittag-Leffler-type functions in fractional evolution processes" J. Comput. Appl. Math. 118 (2000) 283-299.

[23] V.E. Tarasov, "Fractional generalization of Liouville equation" Chaos 14 (2004) 123127; "Fractional systems and fractional Bogoliubov hierarchy equations" Phys. Rev. E 71 (2005) 011102; "Fractional Liouville and BBGKI equations" J. Phys. Conf. Ser. 7 (2005) 17-33; "Transport equations from Liouville equations for fractional systems" Int. J. Mod. Phys. B 20 (2006) 341-354.

[24] V.E. Tarasov, "Fractional generalization of gradient and Hamiltonian systems" J. Phys. A 38 (2005) 5929-5943; "Fractional generalization of gradient systems" Lett. Math. Phys. 73 (2005) 49-58.

[25] V.E. Tarasov, "Fractional variations for dynamical systems: Hamilton and Lagrange approaches" J. Phys. A 39 (2006) 8409-8425.

[26] V.E. Tarasov, G.M. Zaslavsky, "Dynamics with low-level fractionality" Physica A 368 (2006) 399-415.

[27] V.E. Tarasov, "Psi-series solution of fractional Ginzburg-Landau equation" J. Phys. A 39 (2006) 8395-8407.

[28] N. Laskin, G.M. Zaslavsky, "Nonlinear fractional dynamics of lattice with long-range interaction" Physica A 368 (2005) 38-54.

[29] V.E. Tarasov, G.M. Zaslavsky, "Fractional dynamics of coupled oscillators with longrange interaction" Chaos 16 (2006) 023110; "Fractional dynamics of systems with longrange interaction" Commun. Nonlin. Sci. Numer. Simul. 11 (2006) 885-898.

[30] N. Korabel, G.M. Zaslavsky, V.E. Tarasov, "Coupled oscillators with power-law interaction and their fractional dynamics analogues" Commun. Nonlin. Sci. Numer. Simul. 12 (2007) 1405-1417.

[31] M.A. Krasnolelsky, P.P. Zabreiko, E.I. Pustilnik, P.E. Sobolevsky, Integral Operators in Spaces of Integrable Functions (Nauka, Moscow, 1966) sec. 3.4. in Russian

[32] M.A. Krasnolelsky, P.E. Sobolevsky, Dokladi Acad. Nauk SSSR 129 (1959) 499-502.

[33] S.G. Krein, Linear Differential Equations in Banach Space (Nauka, Moscow, 1967) Sec. 1.5. in Russian; and Transl. Math. Monogr. 29, (Amer. Math. Soc. Providence, 1971)

[34] Yu.L. Dalechky, S.G. Krein, Stability of Solutions of Differential Equations in Banach space (Nauka, Moscow, 1970) Sec. 1.2. in Russian; and (Amer. Math. Soc. Providence, 1974) 
[35] S.G. Krein (Ed.), Function analysis 2nd ed. (Nauka, Moscow, 1970) in Russian

[36] V.P. Maslov, Operational Methods (Mir, Moscow, 1976) Sec.3.4.

[37] M.V.Karasev, V.P. Maslov, Nonlinear Poisson Brackets. Geometry and Quantization. (American Mathematical Society, New York, 1993) Appendix 1.

[38] Y. Luke, The Special Functions and Their Approximations (Academic Press, New York, 1969) Volume 1, Chapter 6.

[39] Yu.A. Brichkov, A.P. Prudnikov, Integral transformations of Generalized Functions (Nauka, Moscow, 1977) in Russian

[40] F.A. Berezin, M.A. Shubin, The Schroedinger Equation (Kluwer, Dordrecht, 1991). Sec 5 .

[41] F.A. Berezin, "Non-Wiener functional integrals" Theor. Math. Phys. 6(2) (1971) 194212; "About one representation of operators by functionals" Trudi Moskovskogo Matematicheskogo Obschestva 17 (1967) 117-196.

[42] N.L. Balazs, B.K. Jennings, "Wigner's function and other distribution functions in mock phase spaces" Phys. Rep. 104 (1984) 347-391.

[43] R.F.V. Anderson, "The Weyl functional calculus" J. Funct. Anal. 4 (1969) 240-267; "On the Weyl functional calculus" J. Funct. Anal. 6 (1970) 110-115.

[44] V.E. Tarasov, "Quantization of non-Hamiltonian and dissipative systems" Phys. Lett. A 288 (2001) 173-183; "Weyl quantization of dynamical systems with flat phase space" Moscow University Physics Bulletin, 56/6 (2001) 5-10; "Quantization of nonHamiltonian systems" Theor. Phys. 2 (2001) 150-160; "Path integral for quantum operations" J. Phys. A 37 (2004) 3241-3257.

[45] V.E. Tarasov, Mathematical Introduction to Quantum Mechanics (MAI, Moscow, 2000) in Russian

[46] V.E. Tarasov, Quantum Mechanics. Lectures on Theory Foundation 2nd ed. (Vuzovskaya kniga, Moscow, 2005) in Russian

[47] S. Momani, S.B. Hadid, "Lyapunov stability solutions of fractional integrodifferential equations" Int. J. Math. Math. Sci. 47 (2004) 2503-2507.

[48] S.B. Hadid, J.G. Alshamani, "Liapunov stability of differential equations of noninteger order" Arab J. Math 7 (1986) 5-17.

[49] Y.Q. Chen, K.L. Moore, "Analytical stability bound for a class of delayed fractionalorder dynamic systems" Nonlinear Dynamics 29 (2002) 191-200.

[50] T.D. Khusainov, "Stability analysis of a linear-fractional delay system" Differ. Eq. 37 (2001) 1184-1188.

[51] D. Matignon, "Stability result on fractional differential equations with applications to control processing" In: IMACS - SMC Proceeding, Lille, France, 1996, pp. 963-968. 